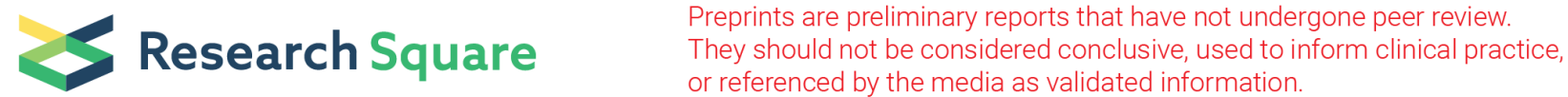

\section{Long-Term Survival in Endoscopic Resection Followed By Additional Chemoradiotherapy For Early-Stage Esophageal Cancer Compared to Esophagectomy: A Systematic Review and Meta- Analysis}

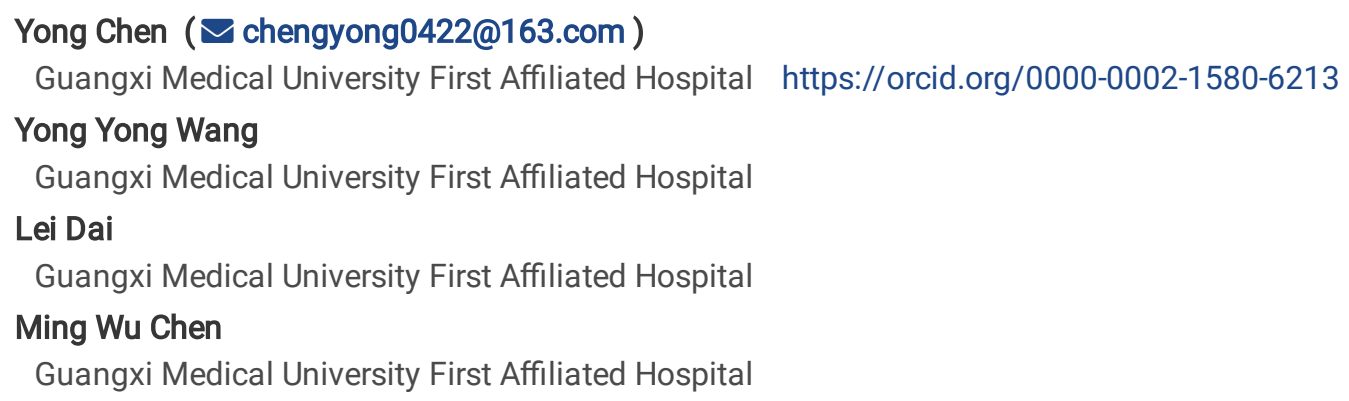

Research

Keywords: Esophageal neoplasms, Endoscopic resection, Adjuvant therapy, Esophagectomy, Chemoradiotherapy, Meta-analysis

Posted Date: November 16th, 2021

DOI: https://doi.org/10.21203/rs.3.rs-1047251/v1

License: (우 (i) This work is licensed under a Creative Commons Attribution 4.0 International License. Read Full License 


\section{Abstract}

Background囚The aim of this study went to systematically evaluate the efficacy of the endoscopic resection followed by adjuvant chemoradiotherapy for early-stage esophageal cancer compared with esophagectomy, and provide reference for clinical research and practice.

Methods: we searched the Cochrane Library, PubMed, Embase, Web of science, CNKI database on line, and collected all of randomized cohort studies and retrospective cohort studies. The quality of the study was evaluated with Cochrane's quality standards, and statistical analysis was performed using STATA version 12.0 and Revman 5.3 software.

Results: A total of 10 studies involving 924 (210 vs 714) patients were included for analysis in our study. long term effect: overall survival rate, disease-free survival rate, recurrence and metastasis rate of esophagectomy group were better than those of the

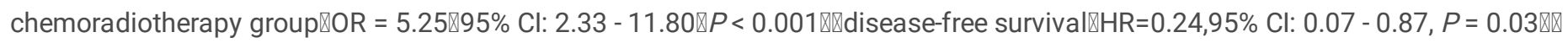

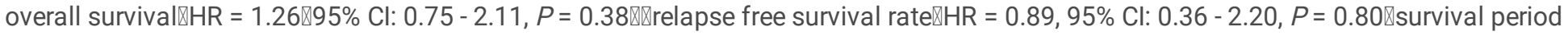
results showed no significant difference $(P \otimes 0.05)$.

Conclusions: Similar outcomes are founding regarding overall survival and relapse free survival rate between the two groups. Meanwhile, chemoradiotherapy may be provided for patients with high-risk of relapse or unable to tolerate surgery. Therefore, chemoradiotherapy is not replaced surgery totally. Further randomized trials are needed to validate the conclusion.

\section{Background}

Esophageal cancer, one of the most common types of malignancy [1]. And it is of the worst harmful digestive tumors with highly aggressive and poor outlook. The 5-year complete and thorough survival rate is less than 25\% [2]. Recently. Endoscopic resection (ER) has been made an accepted option for early-stage superficial esophageal cancer [3]. These early-stage cancers present the best opportunity for a cure at diagnosis, which can affect a long-term survival, about 85\% 5-year survival rate [4]. Monig et al studies [5] reported that the T1a stage lymph node metastasis rate in esophageal squamous cell carcinoma is $0 \%-13 \%$, and the T1b stage lymph node metastasis rate in the superficial submucosa (SM1) is $8 \%-26.5 \%$, and the risk of lymph node metastasis can increase to $22 \%$ $61 \%$ when infiltrating into the submucosal layer (SM2) and below. Shen et al [6] has been proposed that tumor differentiation, vascular invasion and depth of tumor invasion are risk factors for lymph node metastasis. In view of this, it is necessary to add adjuvant chemoradiotherapy (CRT) or esophagectomy after ER.

For a long time, for $\mathrm{cT} 1 \mathrm{a} / \mathrm{bNOM0}$ early esophageal cancer, esophagectomy combined with lymph node dissection has been the standard treatment. Although it has a reliable long term treatment effect, but Patients usually have to bear the risk of postoperative complications and quality of life damage, according to oshi Saeki in a retrospective analysis [7], a total of 34 patients with early-stage esophageal cancer were included. During the 87 month follow up period, no recurrence and metastasis were found; 3 and 5 years of the overall survival (OS) rates were $97.4 \%$ and $89.9 \%$, respectively, postoperative complication was $18 \%$ and $10 \%$, in addition, the overall effect of this treatment is very dependent on the experience of the surgical team and the patient's physical condition.

Different from the features of esophagectomy, such as greater trauma and higher risk of postoperative complication. CRT after ER has become another treatment option. According to a multi-center, single-arm prospective study by Keiko Minashi [8], for the enrollment of 176 patients, the pathological stage was T1b (SM1-2) NOM0, the metastasis recurrence rate was 8.5\%, the 3-year progression-free survival (PFS) rate was $89.7 \%$, and the 3-year OS rate was $90.7 \%$. The long-term treatment effect is considered to be equivalent to surgery, but this study did not include surgical patients, so that the above are only unilateral results.

However, the current researches are mostly retrospective, single center and small sample clinical studies. Therefore, there is still a need for further investigation on the pros and cons of the two treatment methods. This study intends to use meta-analysis methods to comprehensively evaluate the long-term effect of CRT compared with esophagectomy after ER, in order to provide evidence-based medicine for the scientific management of early esophageal cancer.

\section{Methods}

\section{Literature search}


We performed a comprehensive systematic literature search of electronic databases including PubMed, Embase, Web of science, Cochrane Library, and CNKI, from inception to September 2020 to identify all controlled trials and observational studies. The search terms and relative variants were as follows: "esophageal neoplasms", "esophageal cancer", "esophagectomy", "chemoradiotherapy", "chemoradiation", "radio chemotherapy", "esophagoscopy", "endoscopic mucosal resection" and logical combination of these terms using the Boolean operators "AND" and "OR". Present in the title, abstract. hand-searched to identify more studies that may have been missed in the initial search following the reference list of the studies identified.

\section{Inclusion and exclusion criteria}

All potentially relevant articles were considered suitable for enrollment in the analysis using the following inclusion criteria: All eligible patients had esophageal cancer with pT1a-pT1b; studies that examined esophagectomy and additional CRT follow ER; randomized or non-randomized cohort studies; sufficient data of estimated odds ratios (ORs)/weighted mean difference (WMD) and 95\% confidence intervals (Cls). The exclusion are as follows: studies that were not compared or case report; incomplete literature and overlapped studies.

\section{Data extraction}

Two reviewers independently scanned the formal full-length publications of studies which met the inclusion criteria previously described. Discrepancies were mutually resolved through discussion with a third reviewer. Outcomes assessed from the studies included OS, any patterns of recurrence (locoregional recurrence and/or distant metastases and/or locoregional recurrence plus distant metastases). Locoregional recurrence was defined as relapse at the primary site, including the local incision sites or in regional lymph nodes. Distant metastases were defined as distant lymph node sites or distant organs involvement of lung, liver, bone and subcutaneous tissue.

\section{Quality assessment}

For evaluate quality of included studies, randomized controlled trial (RCT) and observational studies were respectively assessed by Cochrane handbook for systematic reviews of interventions (version 5.1.0) and the modified Newcastle-Ottawa Scale (NOS) [9, 10], All trials that fit the inclusion criteria were identified for full review by 2 reviewers. Each reviewer independently selected trials for inclusion in the review. Any disagreement between the 2 extracting authors was resolved by consensus. If consensus among the 2 reviewers cannot be reached after discussion, a third author was deferred to for arbitration and consensus.

\section{Statistical analysis}

Effect sizes for variables were calculated and estimated as standardized mean difference (SMD) for continuous outcomes, and odds ratio $(\mathrm{OR})$ or risk difference $(\mathrm{RD})$ for dichotomous outcomes with $95 \%$ confidence interval $(\mathrm{Cl})$. Heterogeneity among studies was assessed by the $R^{2}$ statistics and Cochran Chi-square test. A random effect model was used for substantial heterogeneity $(R \nabla 50 \%)$ and a fixed effect model in the absence of significant heterogeneity $(R \otimes 50 \%)[11,12]$. When a study included medians and interquartile ranges, we calculated the mean \pm standard deviation (SD) using a method proposed by Hozo et al [13]. All the statistical analyses were performed by using Review Manager (RevMan)v5.3 (the Cochrane Collaboration, Oxford, United Kingdom). The endpoints of this systematic review were divided into primary and secondary result measures. Primary measures: recurrence and metastasis rate. Secondary measures included: OS rate, relapse free survival (RFS) and disease-free survival (DFS).

\section{Risk of bias assessment}

Publication bias was tested using the Funnel plots tests, Egger's and Begg's tests. If either $P$ value was $\llbracket 0.05$, we assumed that there was no significant publication bias $[14,15]$. Data analyses were conducted by using STATA 13.0 (Stata Corporation, College Station, TX), and took $P \leq 0.05$ as statistically significant.

\section{Results}

\section{Study selection and characteristics}

Our search strategy yielded a total of 40 publications suitable for full-text screening. These were screened according to the inclusion criteria to leave a final selection of 10 [16-25]. Fig. 1. illustrates the retrieval process. Of the 10 studies that met the inclusion criteria, 6 were conducted in Japan. The total number of subjects was 924, in which is most of the esophageal squamous cell carcinoma (ESCC), endoscopic submucosal dissection (ESD) as a primary treatment. The chemotherapy regimen included 5-fluorouracil and cisplatin. 1 
was an RCT, based on the scoring system used (score $\geq 5$ ). We conducted NOS on the Non-RCT studies, with a score of 5-8. The baseline characteristics of the selected studies are summarized in Table 1.

Table 1

Study characteristics

\begin{tabular}{|c|c|c|c|c|c|c|c|c|c|}
\hline Author & $\begin{array}{l}\text { Publication } \\
\text { year }\end{array}$ & Country & $\begin{array}{l}\text { Study } \\
\text { type }\end{array}$ & $\begin{array}{l}\text { Sample } \\
\text { size }\end{array}$ & T stage & Pathology & Treatment regimen & $\begin{array}{l}\text { Dose of } \\
\text { RT }\end{array}$ & NOS \\
\hline Tanaka & 2019 & Korea & $\operatorname{Re}$ & 52 & $\mathrm{~T} 1 \mathrm{~b}$ & SCC & ER+CRT/ER+surgery & $>50$ & 7 \\
\hline Lorenzo & 2019 & France & $\mathrm{Re}$ & 10 & T1 & SCC & ESD+CRT/ESD+surgery & NA & 5 \\
\hline Lu & 2019 & America & $\operatorname{Re}$ & 501 & $\mathrm{~T} 1 \mathrm{~b}$ & $A C$ & $\mathrm{ER}+\mathrm{RT} \pm \mathrm{CT} /$ surgery & NA & 5 \\
\hline Suzuki & 2018 & Japan & $\mathrm{Re}$ & 32 & $\mathrm{~T} 1 \mathrm{~b}$ & $\mathrm{SCC}+\mathrm{AC}$ & ESD+CRT/ESD+surgery & $40 / 50$ & 6 \\
\hline Takeuchi & 2018 & Japan & $\operatorname{Re}$ & 32 & M3 T1b & $\mathrm{SCC}+\mathrm{AC}$ & ER+CRT/ER+surgery & 50.4 & 7 \\
\hline Koterazawa & 2018 & Japan & $\operatorname{Re}$ & 59 & $\mathrm{~T} 1$ & SCC & $\mathrm{ESD}+\mathrm{CRT} / \mathrm{ESD}+$ surgery & $41.4 / 50.4$ & 8 \\
\hline Ohki & 2018 & Japan & $\mathrm{Re}$ & 23 & $\mathrm{~T} 1$ & NA & ESD+CRT/ESD+surgery & NA & 6 \\
\hline Huang & 2018 & China & RCT & 135 & M3 T1b & SCC & $\begin{array}{l}\text { ESD/EMR+CRT/ } \\
\text { ESD/EMR+surgery }\end{array}$ & NA & - \\
\hline Ikeda & 2015 & Japan & $\operatorname{Re}$ & 26 & $\mathrm{~T} 1$ & NA & $\mathrm{ESD}+\mathrm{CRT} / \mathrm{ESD}+$ surgery & $41.4 / 50.4$ & 5 \\
\hline Shimizu & 2004 & Japan & $\operatorname{Re}$ & 55 & $\mathrm{~T} 1$ & SCC & EMR+CRT/surgery & $40-46$ & 6 \\
\hline
\end{tabular}

\section{Recurrence and metastasis}

Analysis included a total of 369 patients from eight studies [16, 17, 19-24]. A statistically significant improvement in recurrence and metastasis rate was observed for esophagectomy when compared to the CRT (OR $=5.25,95 \% \mathrm{Cl} 2.33-11.80, P<0.001)$. No heterogeneity was revealed in the result $\left({ }^{2}=0 \%, P=0.87\right)$ (Fig. 2a).

\section{OS}

Seven studies represented a total of 828 patients, with 653 esophagectomy and 175 controls [16, 17, 20,22-25]. No statistically significant OS difference could be established between the both sides (HR=1.26, 95\% Cl: 0.75-2.11, $P=0.38$ ), with a low between-study heterogeneity $\left({ }^{2}=16 \%, P=0.31\right)$ (Fig. 2b). A fixed-effects model was chosen in the remaining groups because of the minimal heterogeneity.

RFS

Analysis included a total of 213 patients from three studies and was evaluated using a random effects model, 124 in the esophagectomy and 89 in the CRT $[16,23,24]$. No significant difference was established in the comparison between the two treatment protocols ( $\mathrm{HR}=0.89,95 \% \mathrm{Cl}: 0.36-2.20, P=0.80)$ with moderate between-study heterogeneity $\left({ }^{2}=48 \%, P=0.15\right)(\mathrm{Fig} .2 \mathrm{c})$.

\section{DFS}

Two studies consisting of a total of 91 patients, 44 in the esophagectomy and 47 in the CRT [19, 20], reported data on DFS rates. Despite small sample size, the results demonstrated that significant superiority of the esophagectomy over the control group $(\mathrm{HR}=0.24,95 \% \mathrm{Cl}$ : $0.07-0.87, P=0.03)$ with insignificant heterogeneity $\left({ }^{2}=0 \%, P=0.39\right)$ (Fig. $2 \mathrm{~d}$ ).

\section{Publication bias and sensitivity analysis}

Funnel plots for the primary outcome measures are represented in recurrence and metastasis and OS (Fig. 3a and 3b). Both representing no publication bias. Sensitivity analysis was used to determine the robustness of the meta-analysis, as well as to determine the main 
factors that influenced the results. Indicating that there was no single study that influenced the results more than another, and confirming that our data were rational. The results of recurrence and metastasis from Begg's test $(P=0.072)$ and Egger's test $(P=$ 0.649); the result of OS from Begg's test $(P=1.000)$ and Egger's test $(P=0.847)$ showed no statistically significant difference, suggesting a small bias in publication.

\section{Discussion}

The treatment plan for esophageal cancer is mainly determined according to its stage. The treatment of early esophageal cancer is mainly based on radical surgery and endoscopic treatment. The clinical effect of endoscopic resection is equal to that of surgery, with less trauma, fewer postoperative complication, and shorter hospital stay. It can be used as the first choice of treatment. However, for some patient with T1b stage who are at risk of lymph node metastasis, ER cannot accomplish lymph node sampling or dissection, and the tumor may be incompletely removed [26]. Adjuvant therapy is believed to improve long-term survival and reduce recurrence rates. At present, main adjuvant treatment methods are esophagectomy and CRT [27]. However, there are still some controversies. First, in terms of safety and effectiveness, whether CRT can completely replace surgery; second, for pathological high-risk factors (lymph node metastasis, vascular nerve invasion), can radical surgery give patients better long-term benefits? In this meta-analysis, we will focus on analyzing the details of the both sides.

Traditionally, radical esophagectomy for early esophageal cancer is still the standard treatment method, but has had obvious treatment risks. Such as anastomotic leakage, lung infection or stenosis, and even death. Andrew J. Yang [28] Concluded through a retrospective study that a total of 179 patients were enrolled, lung infection and esophageal perforation occurred in the surgical group. However, there were no serious complications in the CRT group; in the surgery group, the local recurrence rate was $1 \%$, the regional recurrence rate was $5 \%$, and the distant metastasis rate was $4 \%$; both local and regional recurrences in the CRT group were $8 \%$, and there was no distant metastasis; there was no significant difference in the 5-year OS rate between the two group $(P=0.405)$. the 10 articles included in our study involved a total of 924 patients with early esophageal cancer, the recurrence and metastasis rate of CRT was much higher than that of the surgery group, reaching 5.25 times (Fig. 2a), also, DFS was also better than CRT in surgery group (HR $=0.24,95 \% \mathrm{Cl}: 0.07$ $0.87, P=0.03)$. Two studies $[19,20]$ believe that surgery can give better disease control for tumors $\geq S M 2$ with lymphatic vascular invasion. Unlike ER, first, esophagectomy can completely remove the lesion and perform lymph node dissection, thereby obtaining a more accurate pathological staging and long-term prognosis judgment; second, due to the lack of accurate pathological staging after ER result in insufficient radiation field exposure. Therefore, two reasons are likely to explain why surgery can achieve better performance. Other studies [29] also hold similar views. In addition, included literature showed that no significant differences were found in the OS and RFS. The results are basically consistent with the retrospective study done by Andrew J. Yang [28]. In 2020, Yung-Kuan Tsou [30] conducted a review study, summarizing and reviewing 3 previous related studies, concluded that patients with a high risk of recurrence are recommended to use surgery. Although we hold similar views, but by including 10 articles, we can obtain more credible conclusions.

This study analyzed the application value of CRT after ER of early esophageal cancer, and agreed that CRT can be the preferred choice for the elderly, poor physical condition or unable to tolerate surgery.

However, there is currently no large-scale randomized controlled trial to confirm these points, and thoracic surgery is expected to come from the multi-center randomized controlled trial that is being conducted in China (Chest 201908, ClinicalTrials.gov number: NCT04135664). Meanwhile, early esophageal cancer should be evaluated by a multidisciplinary team, including the participation of gastroenterology, oncology and thoracic surgeons, strive to select a reasonable treatment plan.

This study still has certain limitations, first, the included studies are basically non-randomized controlled trials, selection bias may be unavoidable. Second, because of trust in the efficacy of traditional surgery, patients with a greater risk of tumor recurrence are more likely to undergo esophagectomy, while patients with a relatively low probability of recurrence may undergo CRT. This difference in subjective choice may be increase the curative effect of CRT. Thereby weakening the curative effect of surgery. Nevertheless, our research results still show that surgical resection has better DFS and less probability of metastasis and recurrence, which highlights the reliability of our results; Similarly, in the included literature, the total number of studies from LU is 501[18], but the number of patients in the surgery group is as high as 477 (78\%). Considering the overall stability of the research results, the relevant research results are not included for overall comparison. This reduces the overall sample size of the study; third, of the 10 articles included in this study, 6 articles are from Japan, and relatively few in Europe and America; fourth, the included articles mainly focus on long-term effects. It does not provide detailed short-term effects, such as postoperative complications, CRT toxicity and so on. Last, due to the limitations of the 
included literature data, this study did not conduct further subgroup analysis on the tumor staging of patients, and failed to clarify the difference in the efficacy of surgery and CRT for specific substages.

\section{Conclusion}

Through this meta-analysis, it is found that for the adjuvant treatment after ER of early esophageal cancer. CRT cannot completely replace esophagectomy, and both are equally important treatments. For patients with tumor invasion $\geq$ SM2 or high risk of lymph node metastasis, radical surgery is recommended; for patients with high risk factors for surgery or intolerance, CRT is the preferred option, but this conclusion still needs to be confirmed by a large sample randomized controlled study.

\section{Declarations}

Acknowledgements Not applicable

Author contributions Project development: YC, MWC. Data collection: YC, YYW, LD. Data analysis: YC, MWC. Manuscript writing/editing: YC, YYW, LD, MWC.

Funding This research did not receive any specific grant from funding agencies in the public, commercial, or not-for-profit sectors.

Availability of data and materials The current study was based on the results of relevant published studies.

Conflict of interest The authors report no conflicts of interests.

Ethical statement All procedures performed in studies involving human participants were in accordance with the ethical standards of the institutional and/or national research committee and with the 1964 Helsinki declaration and its later amendments.

Informed consent For this type of study, formal consent is not required.

Consent for publication All authors have reviewed the manuscript and agreed for the publication.

\section{References}

1. Bray F, Ferlay J, Soerjomataram I et al. Global cancer statistics 2018: GLOBOCAN estimates of incidence and mortality worldwide for 36 cancers in 185 countries. CA Cancer J Clin.2018;68(6):394-424.

2. Abnet CC, Arnold M, Wei WQ. Epidemiology of Esophageal Squamous Cell Carcinoma. GASTROENTEROLOGY. 2018;154(2):360-373.

3. Wani S, Drahos J, Cook MB, et al. Comparison of endoscopic therapies and surgical resection in patients with early esophageal cancer: a population-based study. GASTROINTEST ENDOSC. 2014; 79(2):224-232.

4. Marino KA, Sullivan JL, Weksler B et al. Esophagectomy versus endoscopic resection for patients with early-stage esophageal adenocarcinoma: A National Cancer Database propensity-matched study. J Thorac Cardiovasc Surg. 2018,155(5):2211-2218.

5. Mönig S, Chevallay M, Niclauss $N$ et al. Early esophageal cancer: the significance of surgery, endoscopy, and chemoradiation. Ann $N$ Y Acad Sci. 2018,1434(1):115-123.

6. Shen $W$, Shen $Y$, Tan $L$ et al. A nomogram for predicting lymph node metastasis in surgically resected $T 1$ esophageal squamous cell carcinoma. J THORAC DIS. 2018;10(7):4178-4185.

7. Saeki $\mathrm{H}$, Watanabe M, Mine $\mathrm{S}$ et al. Esophagectomy for superficial esophageal cancer after non-curative endoscopic resection. $J$ GASTROENTEROL. 2015;50(4):406-413.

8. Minashi K, Nihei K, Mizusawa J et al. Efficacy of Endoscopic Resection and Selective Chemoradiotherapy for Stage I Esophageal Squamous Cell Carcinoma. GASTROENTEROLOGY. 2019;157(2):382-390.

9. Stang A. Critical evaluation of the Newcastle-Ottawa scale for the assessment of the quality of nonrandomized studies in metaanalyses. EUR J EPIDEMIOL. 2010;25(9):603-605.

10. Cumpston M, Li T, Page MJ et al. Updated guidance for trusted systematic reviews: a new edition of the Cochrane Handbook for Systematic Reviews of Interventions. Cochrane Database Syst Rev.2019;10:D142.

11. MANTEL N, HAENSZEL W. Statistical aspects of the analysis of data from retrospective studies of disease. $J$ Natl Cancer Inst. 1959,22(4):719-748. 
12. DerSimonian R, Laird N. Meta-analysis in clinical trials. Control Clin Trials. 1986,7(3):177-188.

13. Hozo SP, Djulbegovic B, Hozo I. Estimating the mean and variance from the median, range, and the size of a sample. BMC MED RES METHODOL. 2005,5:13.

14. DerSimonian R, Laird N. Meta-analysis in clinical trials revisited. CONTEMP CLIN TRIALS. 2015; 45(Pt A):139-145.

15. Begg CB, Mazumdar M. Operating characteristics of a rank correlation test for publication bias. BIOMETRICS. 1994;50(4):10881101.

16. Tanaka T, Ueno M, lizuka T et al. Comparison of long-term outcomes between esophagectomy and chemoradiotherapy after endoscopic resection of submucosal esophageal squamous cell carcinoma. DIS ESOPHAGUS. 2019,32(12).

17. Lorenzo D, Barret M, Leblanc S et al. Outcomes of endoscopic submucosal dissection for early oesophageal squamous cell neoplasia at a Western centre. United European Gastroenterol J. 2019; 7(8):1084-1092.

18. Lu DJ, David J, Anderson E et al. Alternative Strategies to Esophagectomy in the Management of T1b Esophageal Adenocarcinoma. INT J RADIAT ONCOL. 2019,105(1):E191.

19. Suzuki G, Yamazaki H, Aibe $\mathrm{N}$ et al. Endoscopic submucosal dissection followed by chemoradiotherapy for superficial esophageal cancer: choice of new approach. RADIAT ONCOL. 2018;13(1):246.

20. Koterazawa Y, Nakamura T, Oshikiri T et al. A comparison of the clinical outcomes of esophagectomy and chemoradiotherapy after noncurative endoscopic submucosal dissection for esophageal squamous cell carcinoma. SURG TODAY. 2018;48(8):783-789.

21. Takeuchi M, Suda K, Hamamoto Y et al. Technical feasibility and oncologic safety of diagnostic endoscopic resection for superficial esophageal cancer. GASTROINTEST ENDOSC. 2018,88(3):456-465.

22. Ohki S, Hikichi T, Yamada L et al. Evaluation of additional treatment after non-curative endoscopic submucosal resection for esophageal cancer. DIS ESOPHAGUS. 2018,31:147-148.

23. HUANG B, WANG S, LIU Z et al. Comparative analysis of endoscopic R0 resection followed by additional chemoradiotherapy for early-stage esophageal cancer compared with esophagectomy: A multi-center study from ECETC. Chinese Journal of Clinical Thoracic and Cardiovascular Surgery. 2018; 25(06):466-470.

24. Ikeda A, Hoshi N, Yoshizaki T et al. Endoscopic Submucosal Dissection (ESD) with Additional Therapy for Superficial Esophageal Cancer with Submucosal Invasion. Intern Med. 2015;54(22):2803-2813.

25. Shimizu Y, Kato M, Yamamoto J et al. EMR combined with chemoradiotherapy: a novel treatment for superficial esophageal squamous-cell carcinoma. GASTROINTEST ENDOSC. 2004,59(2):199-204.

26. Nagami Y, Ominami M, Shiba M et al. The five-year survival rate after endoscopic submucosal dissection for superficial esophageal squamous cell neoplasia. Dig Liver Dis. 2017;49(4):427-433.

27. Park I, Kim YH, Yoon DH et al. Non-surgical treatment versus radical esophagectomy for clinical T1N0M0 esophageal carcinoma: a single-center experience. Cancer Chemother Pharmacol. 2014; 74(5):995-1003.

28. Yang AJ, Choi SH, Byun HK et al. Management of Clinical T1N0M0 Esophageal Cancer. GUT LIVER. 2019;13(3):315-324.

29. Lee HJ, Lee H, Park JC et al. Treatment Strategy after Endoscopic Resection of Superficial Esophageal Squamous Cell Carcinoma: A Single Institution Experience. GUT LIVER. 2015,9(6):714-719.

30. Tsou YK, Lee CH, Le PH et al. Adjuvant therapy for pT1a-m3/pT1b esophageal squamous cell carcinoma after endoscopic resection: Esophagectomy or chemoradiotherapy? A critical review. Crit Rev Oncol Hematol. 2020,147:102883.

\section{Figures}




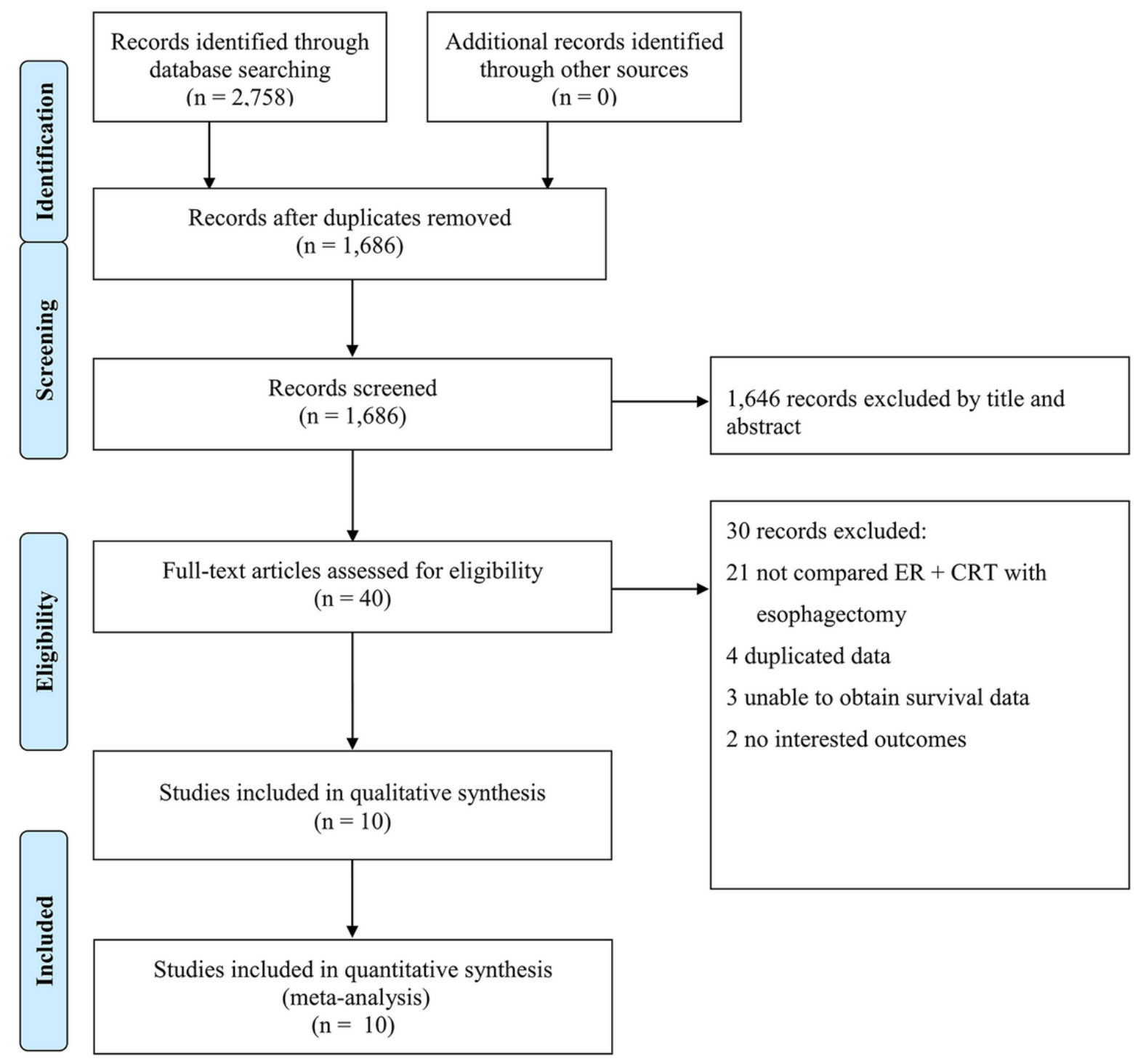

\section{Figure 1}

The Preferred Reporting Items for Systematic Reviews and Meta-analyses (PRISMA) flow chart, detailing the article selection process 
A. Recurrence and metastasis

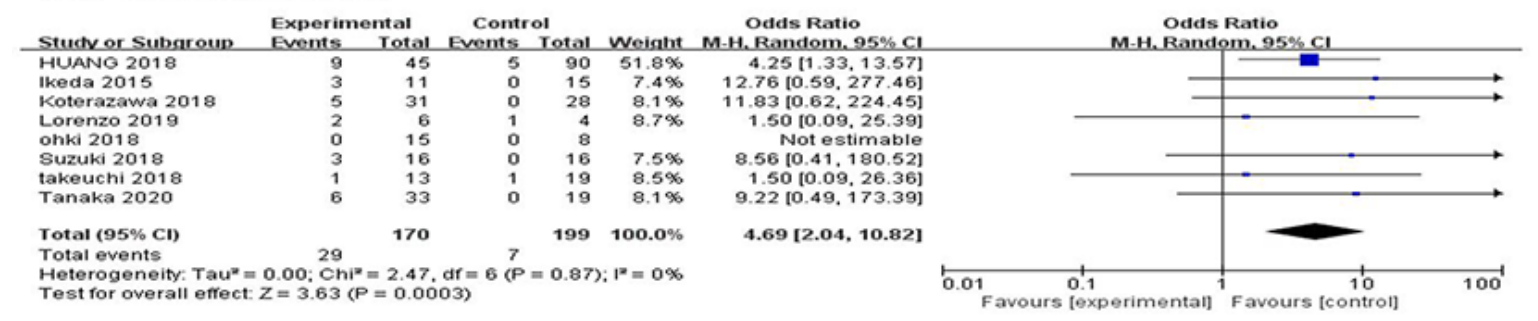

\section{B. OS}

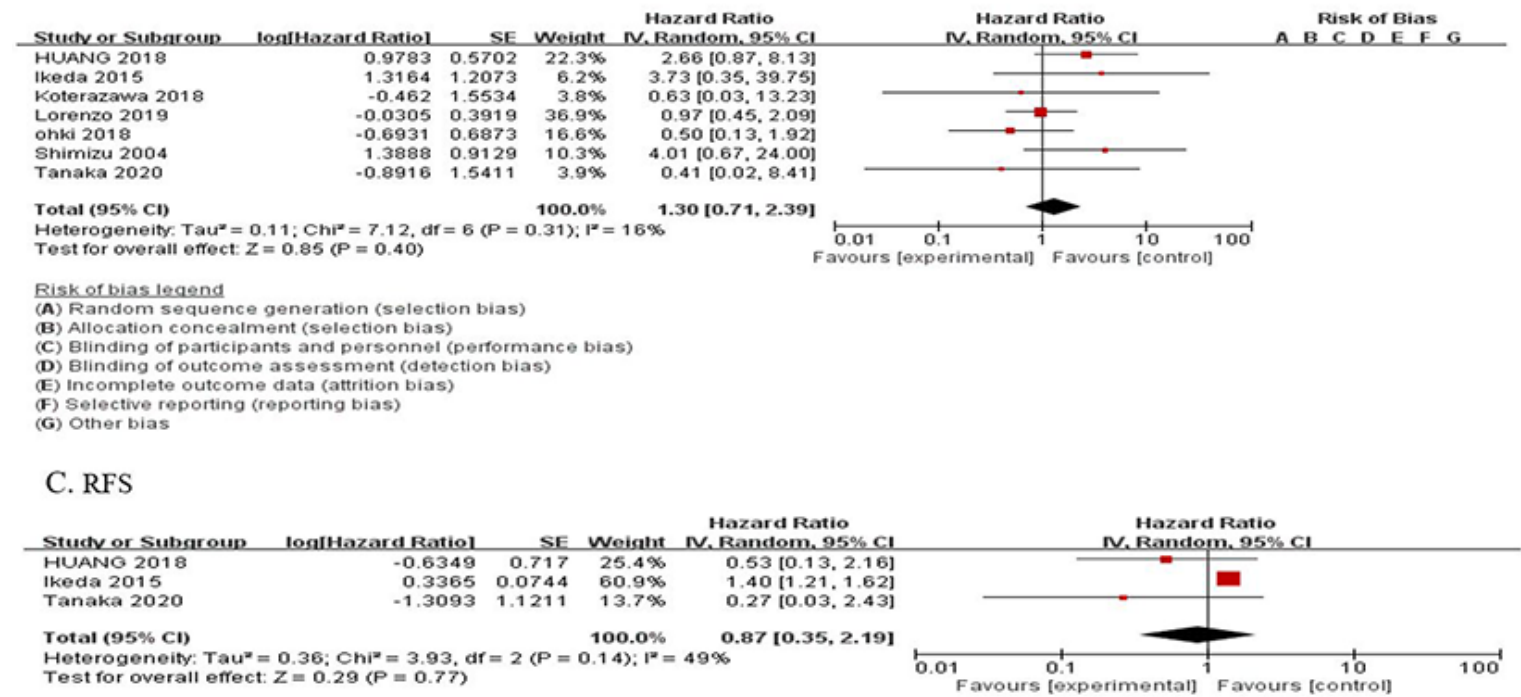

\section{DFS}

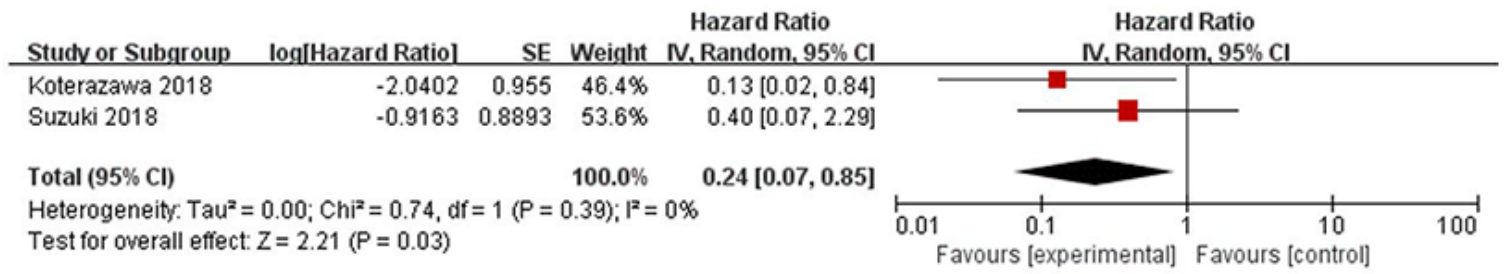

\section{Figure 2}

Forest plot of meta-analysis and cumulative meta-analysis in primary outcomes. a recurrence and metastasis rate, b overall survival (OS), c relapse free survival (RFS), d disease-free survival (DFS).
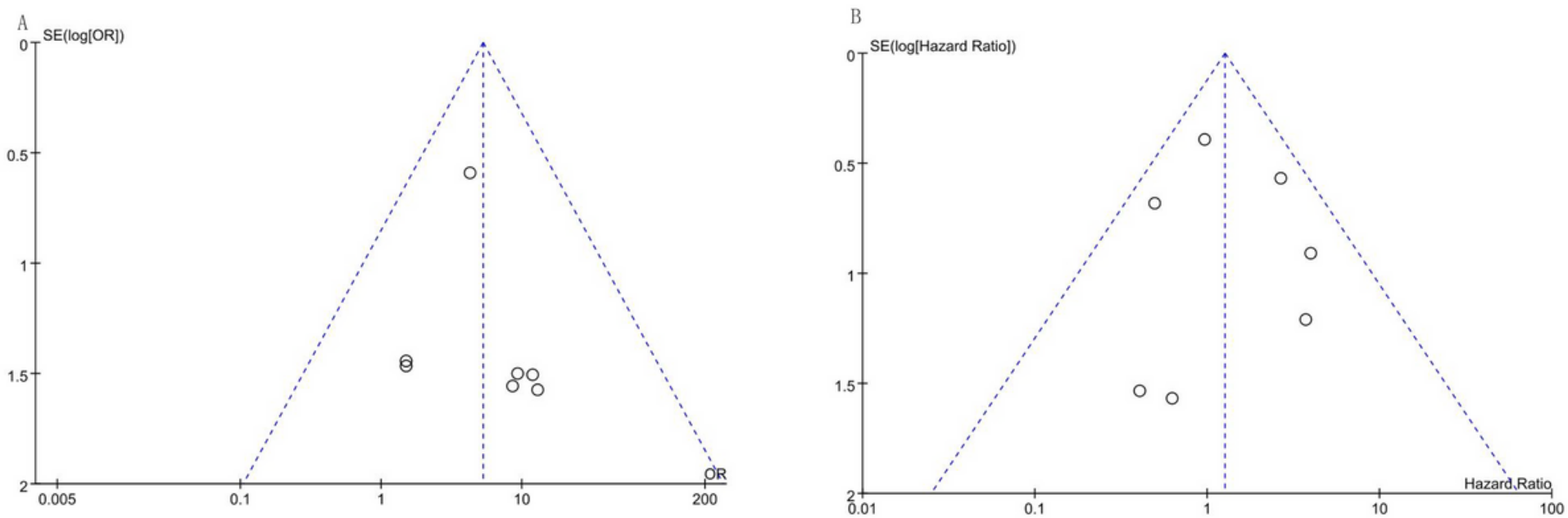


\section{Figure 3}

Funnel plots for primary outcome measures. a recurrence and metastasis, b OS 